Article

\title{
Study on Bubble Cavitation in Liquids for Bubbles Arranged in a Columnar Bubble Group
}

\author{
Peng-li Zhang ${ }^{1,2}$ and Shu-yu Lin ${ }^{1, *}$ \\ 1 Institute of Applied Acoustics, Shaanxi Normal University, Xi'an 710062, China; zhangpl@xust.edu.cn \\ 2 College of Science, Xi'an University of Science and Technology, Xi'an 710054, China \\ * Correspondence: sylin@snnu.edu.cn; Tel.: +86-181-9273-7031
}

Received: 24 October 2019; Accepted: 29 November 2019; Published: 4 December 2019

Featured Application: This work will provide a reference for further simulations and help to promote the theoretical study of ultrasonic cavitation bubbles.

\begin{abstract}
In liquids, bubbles usually exist in the form of bubble groups. Due to their interaction with other bubbles, the resonance frequency of bubbles decreases. In this paper, the resonance frequency of bubbles in a columnar bubble group is obtained by linear simplification of the bubbles' dynamic equation. The correction coefficient between the resonance frequency of the bubbles in the columnar bubble group and the Minnaert frequency of a single bubble is given. The results show that the resonance frequency of bubbles in the bubble group is affected by many parameters such as the initial radius of bubbles, the number of bubbles in the bubble group, and the distance between bubbles. The initial radius of the bubbles and the distance between bubbles are found to have more significant influence on the resonance frequency of the bubbles. When the distance between bubbles increases to 20 times the bubbles' initial radius, the coupling effect between bubbles can be ignored, and after that the bubbles' resonance frequency in the bubble group tends to the resonance frequency of a single bubble's resonance frequency. Fluent software is used to simulate the bubble growth, shrinkage, and collapse of five and seven bubbles under an ultrasonic field. The simulation results show that when the bubble breaks, the two bubbles at the outer field first begin to break and form a micro-jet along the axis line of the bubbles. Our methods and conclusions will provide a reference for further simulations and indicate the significance of the prevention or utilization of cavitation.
\end{abstract}

Keywords: dynamic equation; bubbles; resonance frequency; bubble number; distance

\section{Introduction}

Cavitation is a typical hydrodynamic phenomenon that exists in many fields, such as hydraulic engineering [1], shipping [2], ultrasonic cleaning [3], underwater explosions [4], and the medical industry [5]. There are many reports on single and two cavitation bubble dynamics and the interaction between bubbles near a wall [6-8]. In fact, bubbles in liquids always exist in the form of a bubble group. The shape of bubble groups in liquids is very complicated and the vibration, translation, size, shape, and life of each bubble in a bubble group are not the same. Moreover, there is a weak mutual coupling between the bubbles in a bubble group [9]. It is of a great significance to study the utilization of cavitation and coupling between multiple bubbles [10-12]. Nicolas Bremond et al. [13] have investigated the dynamic behavior of cavitation bubbles on solid surfaces via experiments and numerical simulations. They believed that the pressure generated by each individual bubble should be considered in the Rayleigh-Plesset equation, and that the outer bubble movement period was short. Cui et al. [14] have studied the interaction of two bubbles of the same size in a free-field. An [15] has investigated the dynamic equation of bubbles in a columnar bubble group and analyzed the kinetic 
behavior of bubbles and the characteristics of multi-bubble sonoluminescence. The study of bubble dynamics is beneficial to regulating and controlling the intensity of ultrasonic cavitation. Ohl [16] and Luo Jing [12] have pointed out that the velocity of a bubble micro-jet is in the range $20 \sim 150 \mathrm{~m} / \mathrm{s}$. Tomita et al. [17] have observed through high-speed photography that when small bubbles are near a wall, these small bubbles under the action of a shock wave directly hit the wall, which may cause a certain degree of denudation of the structure. Han et al. [18] have conducted experimental and numerical studies on the interaction of two bubbles in a free field. Ida et al. [19] have found that injecting large bubbles in liquids can inhibit the occurrence and development of cavitation, and their research has mainly discussed the influence of the interactions between bubbles near a wall.

The shape distribution of bubbles in water is very complicated, but with the modulation of ultrasonic waves, bubbles will aggregate into stable special structures such as "bubble grapes", "cone-like bubble structures", "Acoustic lichtenberg figures", and "spherical" structures [20-24]. Studies have shown that the secondary Bjerkens force between bubbles is a major factor in bubble clusters [25-27]. Classical Bjerknes force theory states that two interacting bubbles can only exhibit the following behaviors: if the ultrasonic frequency is between the resonance frequency of two bubbles, the bubbles will repel each other; if the ultrasonic frequency is beyond the resonance frequency of two bubbles, the bubbles will attract each other. Hence, it is very important to learn about the resonance frequency of bubbles. Wang et al. [28] have simulated the coupling of two horizontally arranged bubbles under a free surface. However, the number of bubbles in a bubble group is very large, and the shape of the bubbles is very complicated. In order to analyze coupling effects between bubbles, the spherical and columnar bubble group models are widely used. S. k. Spratt et al. [29] concluded that the radius of bubbles in a champagne glass should be $0.94 \mathrm{~mm}$ according to the Minnaert frequency relationship by monitoring the radial acoustic frequency of bubbles at the bottom of a champagne glass. In fact, there is a weak coupling interaction between bubbles in a bubble group. This coupling effect is a kind of inhibitory effect on bubble growth, oscillation, and collapse; after this effect the resonance frequency of the bubble decreases [30,31].

The resonance frequency of bubbles in a bubble group is an important parameter with which to study the cavitation phenomenon, whether bubbles collapse in an ultrasonic field, and the secondary Bjerkens force between bubbles. However, research on bubble groups has mainly been focused on bubbles near walls or liquid surfaces, and most of these works are experimental studies with few theoretical explanations. There is little literature on the resonance frequency of bubbles in a bubble group. Hence, in this paper, the resonance frequency of bubbles in a columnar bubble group is obtained by linear simplification of the bubbles' dynamic equation. The correction coefficient between the resonance frequency of bubbles in a columnar bubble group and the Minnaert frequency of a single bubble is given. Fluent fluid dynamics software is used to simulate bubble growth, shrinking, and collapse in five- and seven-columnar bubble groups under an ultrasonic field.

\section{Theoretical Model}

If the effect of liquid turbulence is not considered, when the distance between bubbles and the size of the bubbles are much smaller than the wavelength of the ultrasonic waves in a liquid, it can be assumed that the bubbles are in the same ultrasonic field. When the distance between the bubbles is $d_{i j}$, the bubble dynamics equation of the bubble group under the ultrasonic field can be expressed as $[32,33]$

$$
\begin{aligned}
& R_{i} \ddot{R}_{i}+\frac{3}{2} \dot{R}_{i}^{2}=\frac{1}{\rho}\left[\left(p_{0}+\frac{2 \delta}{R_{i 0}}-p_{v}\right)\left(\frac{R_{i 0}}{R_{i}}\right)^{3 k}+p_{v}-p_{0}-\frac{2 \delta}{R_{i}}-\frac{4 u}{R_{i}} \dot{R}_{i}-p_{a} \sin (\omega t)\right] \\
& -\sum_{j=1}^{N} \frac{R_{j}}{d_{i j}}\left[R_{j} \frac{\mathrm{d}^{2} R_{j}}{\mathrm{~d} t^{2}}+2\left(\frac{\mathrm{d} R_{j}}{\mathrm{~d} t}\right)^{2}\right]
\end{aligned}
$$

The last item in the above equation reflects the interaction between cavitation bubbles. $R_{i}$ is the bubble radius at any time, $R_{i 0}$ is the bubble's initial radius, $\rho$ is the liquid density out of the bubble, $p_{0}$ 
is the pressure of the liquid outside the bubble wall, $u$ is the liquid viscosity coefficient, $\sigma$ is the surface tension coefficient, and $p_{v}$ is the gas pressure inside the bubble.

In fact, the movement of the bubbles under the ultrasonic field is a forced vibration. The main parameters that affect the forced vibration of the bubbles include resonance frequency, ultrasonic frequency, and amplitude. In order to investigate the relationship between the resonance frequency of the cavitation bubble and the number of bubbles, the initial radius of the bubble, and the distance between bubbles, Equation (1) has been used as a linear simplification. Assuming that the distance between bubbles in a columnar group is $d$ and that all bubbles have the same initial radius, the bubble we investigate here is the first one. At this time, the distance between any bubble and the first bubble can be expressed as $d_{j 1}=n d$, as shown in Figure 1 .

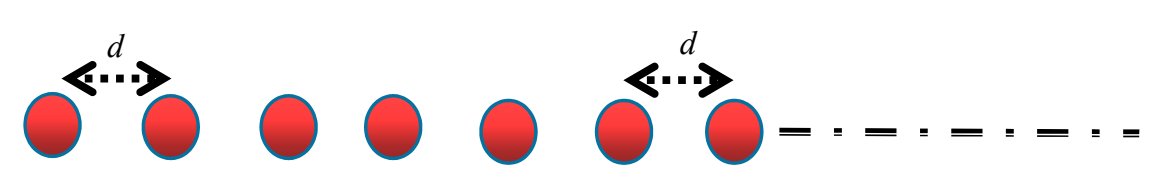

Figure 1. Bubbles in a columnar bubble group; the spacing between the bubbles is $d$.

Hence, the interaction term between bubbles in Equation (1) is simplified as

$$
\sum_{j=1}^{N} \frac{R_{j}}{r_{i j}}\left[R_{j} \frac{\mathrm{d}^{2} R_{j}}{\mathrm{~d} t^{2}}+2\left(\frac{\mathrm{d} R_{j}}{\mathrm{~d} t}\right)^{2}\right]=\sum_{n=1}^{n} \frac{1}{n d} \frac{\mathrm{d}\left(R^{2} \dot{R}\right)}{\mathrm{d} t}
$$

According to Euler's approximation from 1734 [34,35], Equation (2) can be changed to

$$
\sum_{n=1}^{n} \frac{1}{n d} \frac{\mathrm{d}\left(R^{2} \dot{R}\right)}{\mathrm{d} t}=\frac{\mathrm{d}\left(R^{2} \dot{R}\right)}{\mathrm{d} t} \frac{(\ln (n+1)+\xi)}{d}
$$

where $\xi$ is a constant and $\xi \approx 0.577218$. Let $(\ln (n+1)+\xi) / d=A$, substituting (3) into (1), and the following can be obtained.

$$
\left(R+A R^{2}\right) \ddot{R}+\left(\frac{3}{2}+2 A R\right) \dot{R}^{2}=\frac{1}{\rho}\left[\left(p_{0}+\frac{2 \delta}{R}-p_{v}\right)\left(\frac{R_{0}}{R}\right)^{3 k}+p_{v}-p_{0}-\frac{2 \delta}{R}-\frac{4 u}{R} \dot{R}-p_{a} \sin (\omega t)\right]
$$

In the case of small vibrations of the bubble, ignoring the influence of the viscosity coefficient of the liquid

$$
R=R_{0}(1+x) \text { and } x \ll 1, \dot{R}=R_{0} \dot{x}, \ddot{R}=R_{0} \ddot{x}
$$

and

$$
\left(\frac{R_{0}}{R}\right)^{3 \gamma} \approx 1-3 \gamma x, \frac{1}{R} \approx(1-x) \frac{1}{R_{0}}
$$

By substituting (5) and (6) into Equation (4), and considering $x$ to be a small amount, we obtain

$$
\ddot{x}+\left[\omega_{r}^{2}-\frac{1}{\rho\left(R_{0}^{2}+A R_{0}^{3}\right)} p_{a} \sin \left(\omega_{a} t\right)\right] x=\frac{1}{\rho\left(R_{0}^{2}+A R_{0}^{3}\right)} p_{a} \sin \left(\omega_{a} t\right)
$$

In Equation (7), $\omega_{r}$ is the circular resonance frequency of the bubble, and can be expressed as

$$
\omega_{r}^{2}=\frac{1}{\rho\left(R_{0}^{2}+A R_{0}^{3}\right)}\left[3 \gamma\left(p_{0}+\frac{2 \sigma}{R_{0}}-p_{v}\right)-\frac{2 \sigma}{R_{0}}\right]
$$

or as

$$
f_{r}=\frac{1}{2 \pi R_{0}\left(1+A R_{0}\right)^{\frac{1}{2}}}\left[\frac{3 \gamma}{\rho}\left(p_{0}+\frac{2 \sigma}{R_{0}}-p_{v}\right)-\frac{2 \sigma}{R_{0}}\right]^{\frac{1}{2}}
$$


From Equation (9) it is known that the resonance frequency of a bubble in a columnar bubble group is related to the distance between the bubbles and the number of bubbles in the bubble group.

In order to describe the relationship between the bubble resonance frequency of the bubble group and the Minnaert frequency of a single bubble, a correction coefficient $M$ is given to the Minnaert frequency of the single bubble, where $M=f_{r} / f_{0}$, i.e.,

$$
f_{0}=\frac{1}{2 \pi R_{0}}\left[\frac{3 \gamma}{\rho}\left(p_{0}+\frac{2 \sigma}{R_{0}}-p_{v}\right)-\frac{2 \sigma}{\rho R_{0}}\right]^{\frac{1}{2}}
$$

and then

$$
M=\left(\frac{1}{1+A R_{0}}\right)^{\frac{1}{2}}
$$

Equation (11) is the correction relationship between the resonance frequency of the bubble in the spherical bubble group and the single bubble Minnaert frequency.

\section{Study of Resonance Frequency of Bubbles in a Bubble Group}

From Equation (9) it can be seen that the resonance frequency of the bubbles in a columnar bubble group is related to the distance between the bubbles, the number of bubbles in the bubble group, and the initial radius of the bubbles. Hence, we have been able to numerically calculate the relationship between the distance between the bubbles, the number of bubbles, and the resonance frequency of the bubbles. From Equation (9) we obtain Figures 2-4, where the values of the parameters are, respectively, $\rho=999 \mathrm{~kg} / \mathrm{m}^{3}, \gamma=1.33, \sigma=0.072 \mathrm{~kg} / \mathrm{s}^{2}, p_{0}=1 \mathrm{bar}$, and $p_{v}=2250 \mathrm{P}_{\mathrm{a}}$.

The relationship between the number of bubbles and the resonance frequency of the bubbles is shown in Figure 2. It can be seen from the figure that the more bubbles there are, the smaller the resonance frequency of the bubbles. When the bubble number increases, the resonance frequency of the bubbles changes quickly. When the number of bubbles changes from two to twenty-five the change in bubble resonance frequency is most obvious; the resonance frequency changes from $135.8 \mathrm{kHz}$ to $117.8 \mathrm{kHz}$, which is a reduction of about $18 \mathrm{kHz}$. After this, the number of bubbles increases and the resonance frequency tends towards a certain value.

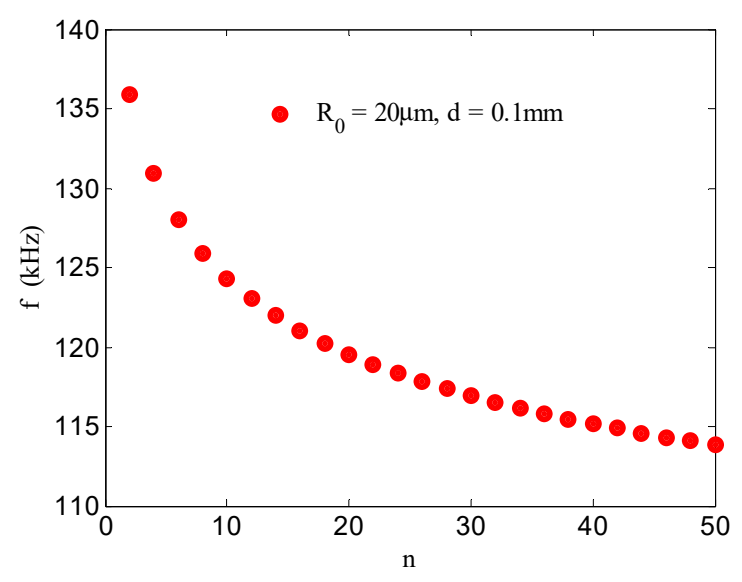

Figure 2. Relationship between the number of bubbles and the resonance frequency of each bubble in a columnar bubble group.

The relationship between the distance between bubbles and the resonance frequency is given in Figure 3, where the initial radius of each bubble is $20 \mu \mathrm{m}$ and the number of bubbles is five. It can be seen from the figure that when the distance between the bubbles increases and the interaction between the bubbles weakens, after that the resonance frequency of the bubbles increases and finally tends towards a fixed value which is the Minnaert frequency of a single bubble. When the distance between 
bubbles increases to 20 times the initial radius the interaction between the bubbles is almost negligible. According to Figures 2 and 3, it can be concluded that, with regard to the bubbles' initial radius, when the distance between bubbles increases to 20 times the initial radius, or is equal to twenty bubbles in a columnar, the interaction between the bubbles is very weak and can be ignored.

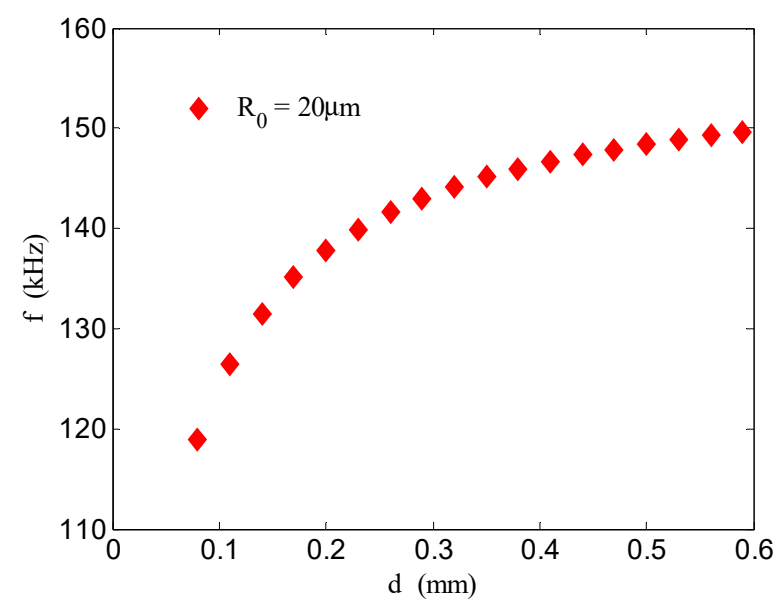

Figure 3. Relationship between the distance between bubbles and the bubble resonance frequency in a columnar bubble group, $n=5$.

Figure 4 shows that the resonance frequency of a bubble is affected by the initial radius of the bubbles, the distance between bubbles, and the number of bubbles in the bubble group. It can be seen from Figure $4 \mathrm{a}, \mathrm{b}$ that when the space between bubbles increases, the inhibitory effect between bubbles becomes weak and the bubble resonance frequency increases until the coupling effect disappears and finally returns to a single bubble resonance frequency. In Figure 4 it can also be observed that for different initial radii and different numbers of bubbles after twenty bubbles, the coupling effect between the first bubble and others becomes very weak. Additionally, when the distance between bubbles increases to 20 times the initial radius of the bubbles, the interaction between bubbles can be neglected. When the initial radius of the bubbles is smaller and the bubbles are closer to each other the coupling force between bubbles will be stronger.
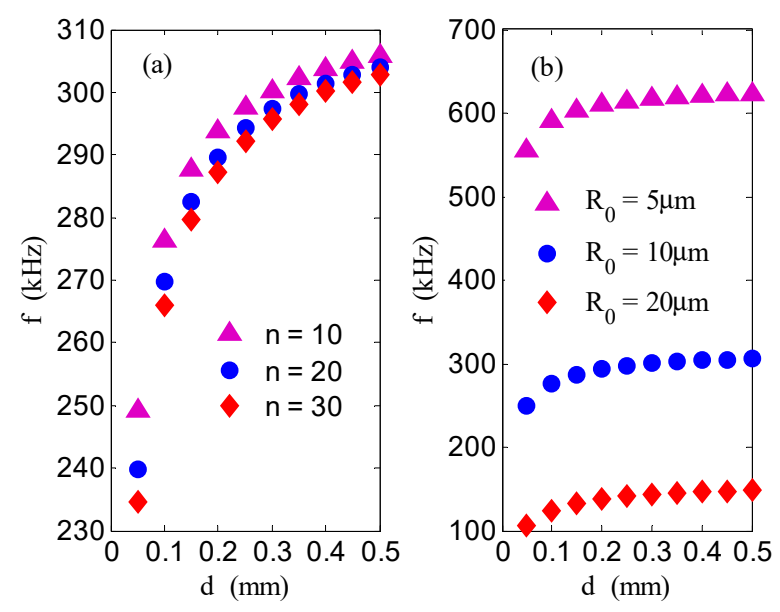

Figure 4. Relationship between resonance frequency and different number of bubbles as well as different initial radius of bubbles. In (a), correspond to different numbers of bubbles (respectively, 10, 20, and 30, where $R_{0}=10 \mu \mathrm{m}$ ) and in (b) correspond to the different number of bubbles' initial radii (respectively, $5 \mu \mathrm{m}, 10 \mu \mathrm{m}$, and $20 \mu \mathrm{m}$, where $n=10$ ). 


\section{Fluent Analysis}

Bremond et al. [36] have used a CCD (charge-coupled device camera) to capture morphological changes of the growth and collapse of single bubbles, two-bubble groups, and five-bubble groups. Their experimental results show that morphological changes during a multi-bubble collapse vary with the spacing of bubbles. At the same time, they found that the outer bubble movement period is shorter and that the jet of outer bubbles is completed first. This method can control the cavitation bubbles' location, but cavitation bubbles can only form near the wall's surface; in this case, the influence of the wall's surface on cavitation bubble collapse cannot be ignored. Due to the influence of fluid properties, the micron scale of bubbles, instantaneous collapse, and other factors, it is impossible to accurately control bubbles in the bubble group, leading to the failure of bubble research under many conditions through experiments. With the development of computational fluid dynamics, numerical simulations have played an increasingly important role in the study of bubble dynamics. Simulation of the cavitation process by Fluent software can show the micro-bubble growth process and the rapid collapse process, as well as reflect the mutual coupling process of bubbles in the bubble group and the non-spherical change of the bubbles.

It has been pointed out that under a weak coupling effect between bubbles, bubbles maintain the shape of the hemisphere most of the time without contact and fusion, but, when the bubbles collapse, they form an interfacing jet [12]. We simulated the growth and collapse process of bubbles for five and seven bubbles set in a line using Fluent software with an initial radius of $30 \mu \mathrm{m}$ and a distance of $200 \mu \mathrm{m}$ between bubbles. It can be found from Equation (9) that when the number of bubbles is five the resonance frequency of the bubbles is $89.8 \mathrm{kHz}$ and that when the number of bubbles is seven the resonance frequency of the bubbles is $88.4 \mathrm{kHz}$. Using a volume of fluid (VOF) 2D axisymmetric analysis has the advantage of easy realization, small computational complexity, and high precision, and traces the volume of fluid in the grid, not the motion of the fluid particles [37]. For the simulation domain, shown in Figure 5, the calculation field is $3 \mathrm{~mm} \times 6 \mathrm{~mm}$, the number of cells is 253,000 , the number of nodes is 254,331 , and the calculation step is $0.01 \mu \mathrm{s}$. The liquid medium is water and the gas inside the bubble is nitrogen. In the process of simulation and calculation, the ultrasonic field is driven from the left side, for which $P_{a}=p_{a} \sin (2 \pi f t)-p_{a}$ is the amplitude of the ultrasonic pressure and $f$ is the ultrasonic frequency. The right side is the free interface and the outlet pressure is the hydrostatic pressure, which is an atmospheric pressure. The ultrasonic frequency $f$ is $26 \mathrm{kHz}$, which is smaller than the resonance frequency of the bubble, and the ultrasonic pressure amplitude $p_{a}$ is 1.2 bar. Then we obtained Figures 6-9.

Figures 6 and 8 show that the bubble has grown well and remained spherical under the ultrasonic field. Due to the mutual restriction of the bubbles during the growth process, the two bubbles at the outer field can reach maximum volume first in the expansion process, and the maximum volume is larger than the inner bubble volume. In time, all bubbles begin to shrink simultaneously. In the process of shrinkage, the outer bubbles shrink slightly faster, becoming an ellipsoid first, and can maintain their ellipsoid shape while continuously shrinking. When contracted to a certain extent, the outer two bubbles begin to collapse first, forming a micro-jet and directing it towards the bubbles back and along the axial center of the wire-packing group to break the bubbles one by one until the group is completely torn open. At this time, due to the sharp change in the pressure gradient in the liquid domain, the intermediate bubbles are stretched from an ellipsoid to a gourd shape and stretched to split into two bubbles. Next, the micro-jet generated by the edge bubbles continues to break down the second and third bubbles in turn until all the bubbles are completely torn apart. Before the breakdown of the intermediate bubbles, because of the close distance between the bubbles, it is possible for the intermediate bubbles to fuse. The numerical simulation results are in good agreement with the experimental conclusions as given in Figure 10 by Bremond [36]; we replicate Figure 10 here again. 


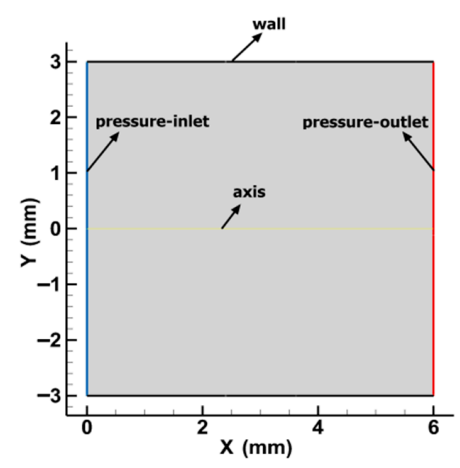

(a)

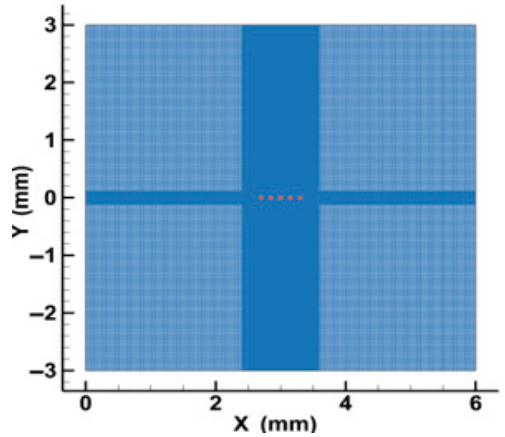

(b)

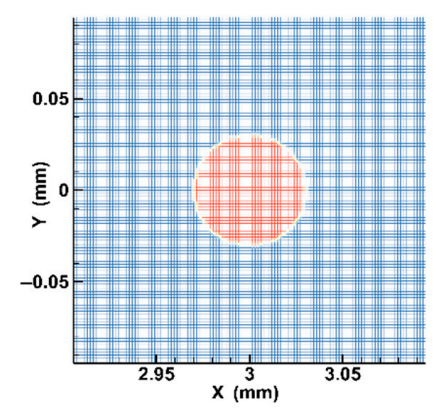

(c)

Figure 5. Simulation domain and meshing: $(\mathbf{a}, \mathbf{b})$ show the calculation of the domain and the linear bubble in the liquid and (c) shows the third bubble and mesh in the domain.

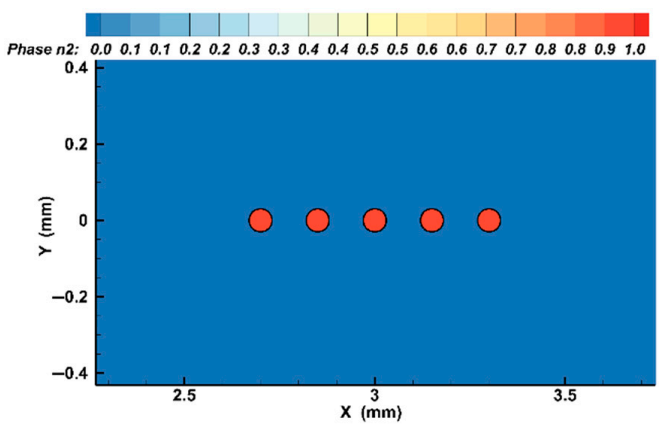

(a) $\mathrm{t}=0$

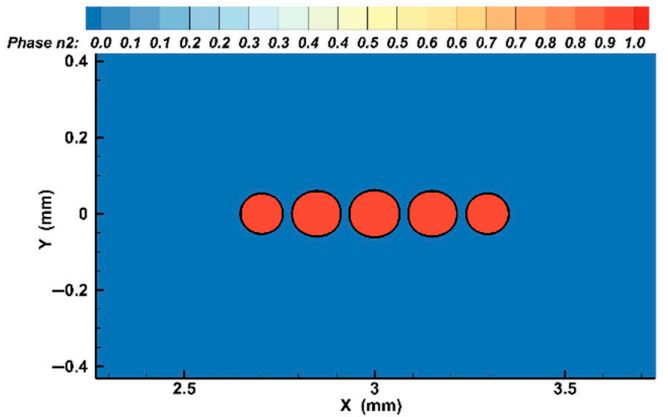

(c) $\mathrm{t}=16 \mu \mathrm{s}$

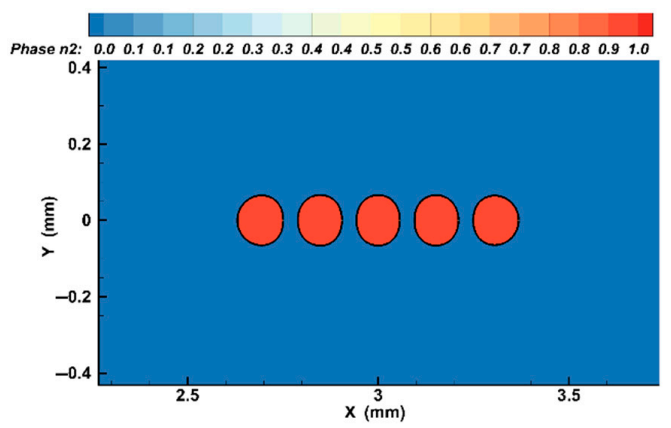

(b) $\mathrm{t}=8 \mu \mathrm{s}$

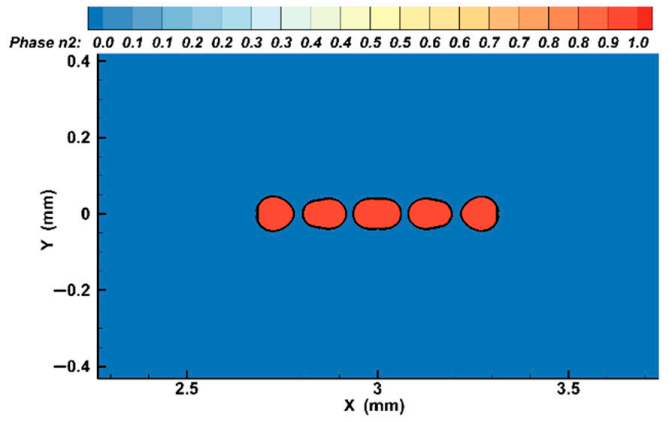

(d) $\mathrm{t}=20 \mu \mathrm{s}$

Figure 6. Cont. 


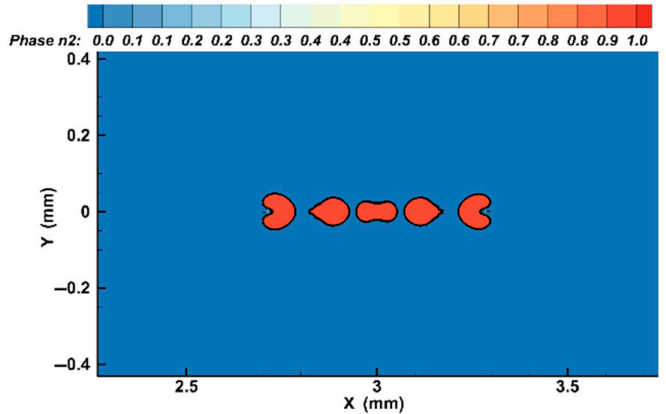

(e) $\mathrm{t}=30 \mu \mathrm{s}$

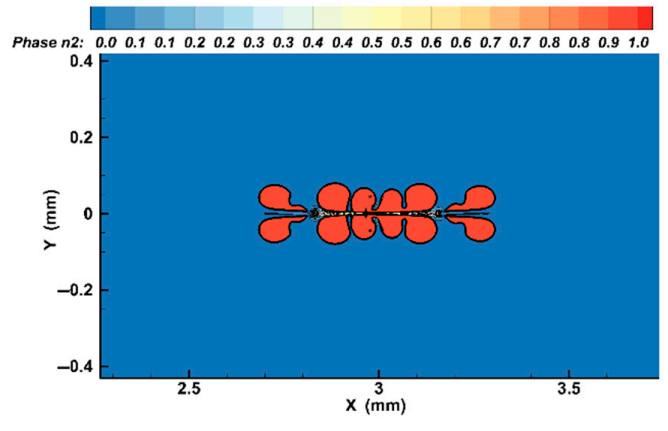

(f) $\mathrm{t}=35 \mu \mathrm{s}$

Figure 6. Bubble volume fraction in the columnar bubble group; $n=5$.

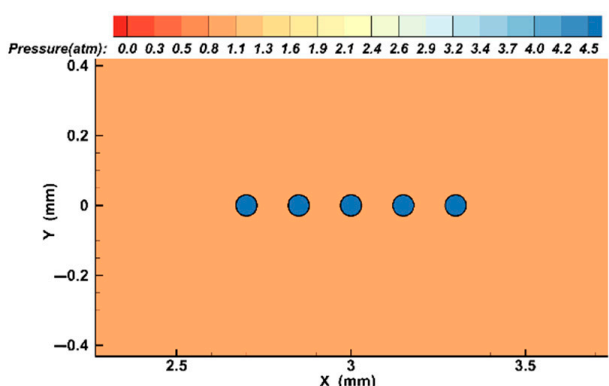

(a) $\mathrm{t}=0$

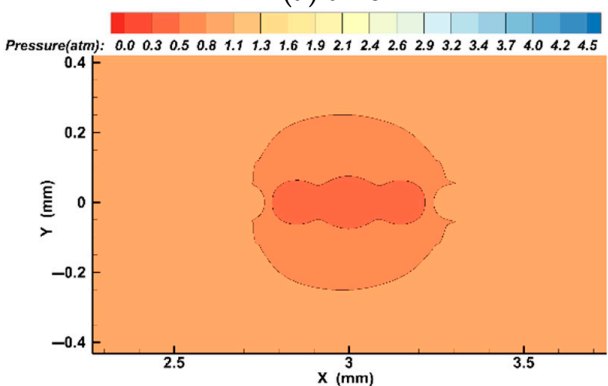

(c) $\mathrm{t}=16 \mu \mathrm{s}$

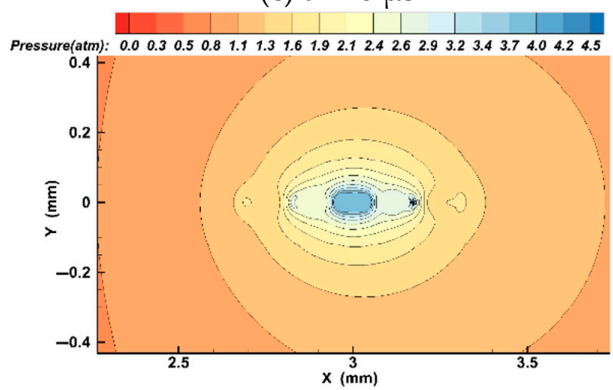

(e) $\mathrm{t}=30 \mu \mathrm{s}$

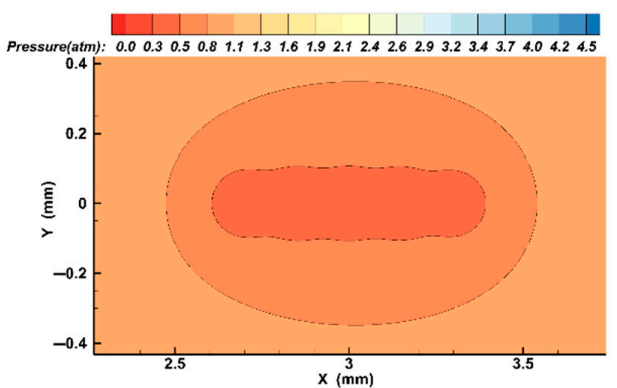

(b) $\mathrm{t}=8 \mu \mathrm{s}$

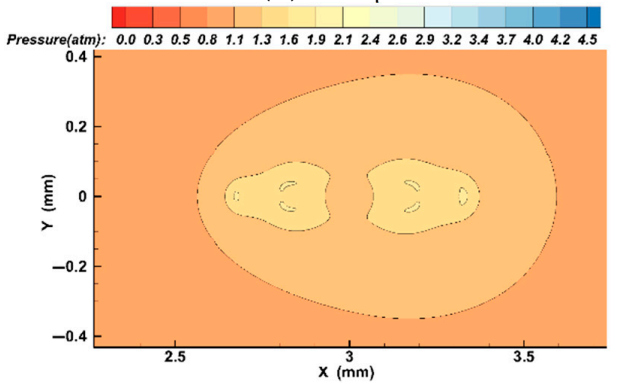

(d) $\mathrm{t}=20 \mu \mathrm{s}$

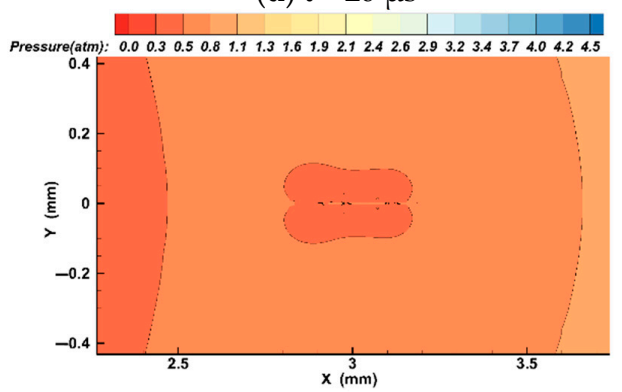

(f) $\mathrm{t}=35 \mu \mathrm{s}$

Figure 7. Pressure distribution in the bubble group; $n=5$. 


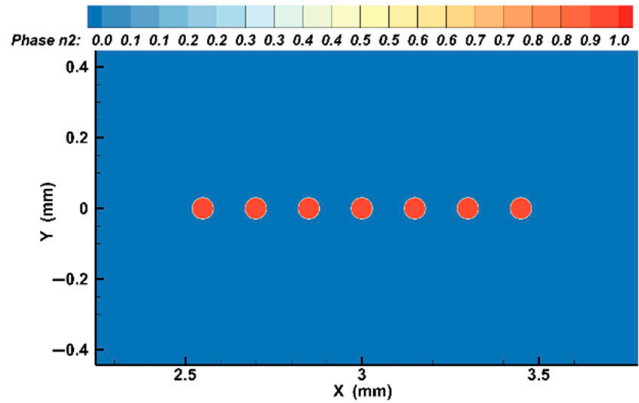

(a) $\mathrm{t}=0$

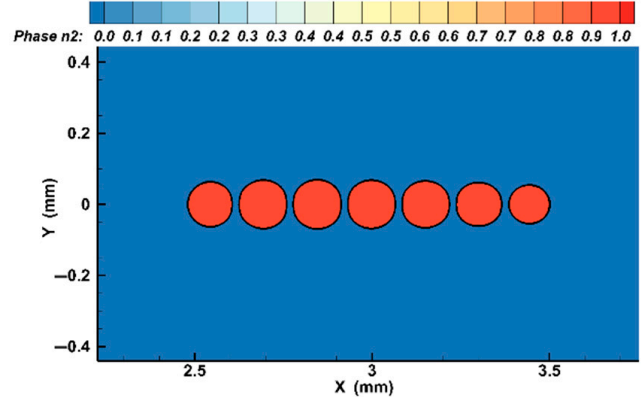

(c) $\mathrm{t}=18 \mu \mathrm{s}$

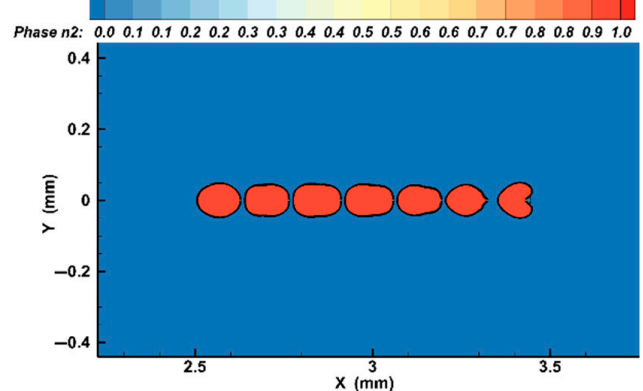

(e) $\mathrm{t}=24 \mu \mathrm{s}$

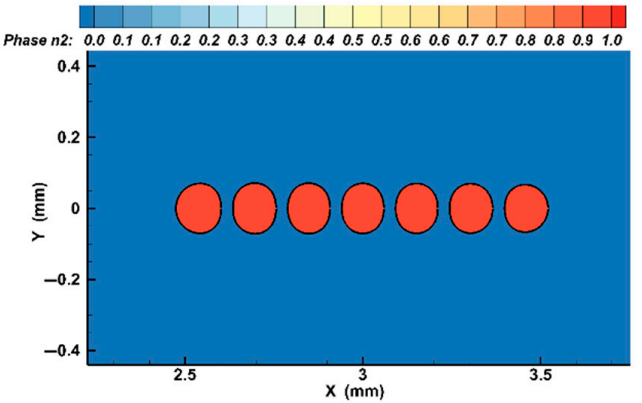

(b) $\mathrm{t}=10 \mu \mathrm{s}$

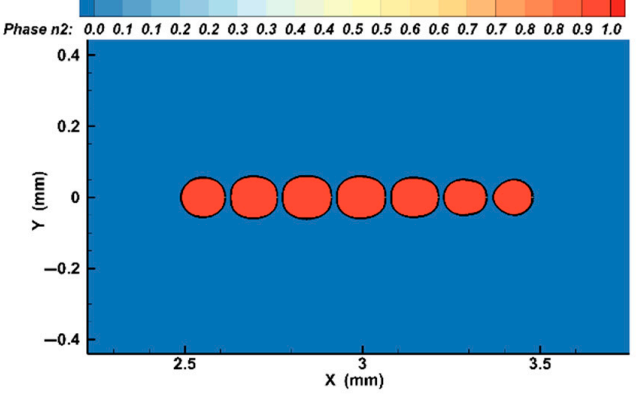

(d) $\mathrm{t}=21 \mu \mathrm{s}$

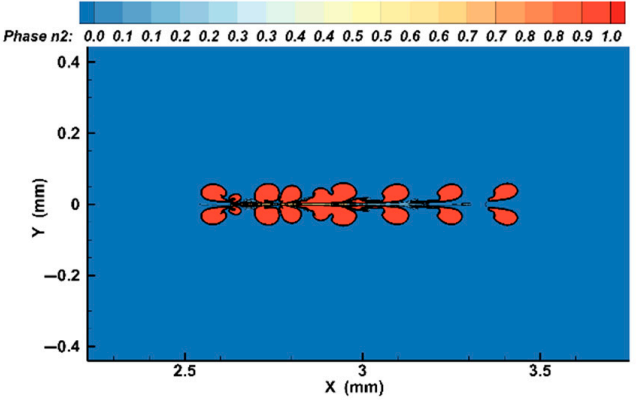

(f) $t=32 \mu \mathrm{s}$

Figure 8. Bubble volume fraction in the columnar bubble group; $n=7$.

Figures 7 and 9 show that the pressure inside the bubbles decreases sharply during bubble expansion and that when the bubble expands to the maximum volume, the pressure inside the bubble is only 0.41 bar; in the period of bubble shrinkage and collapse, the pressure inside the bubble rises sharply. When the bubble collapses, the pressure inside the bubble reaches its maximum value of about 1.82 bar. After the bubble collapses in Figure 9f, the pressure inside and outside the bubble tends to be almost the same. Due to the micro-jet generated by the bubble collapse, the pressure on the jet line is significantly greater than in other locations. In addition, on account of the mutual coupling between the bubbles, the resonance frequency of the bubbles is reduced, and under the same acoustic parameters a large number of bubbles collapse sooner and more easily. The pressure inside the bubble also changes more sharply. 


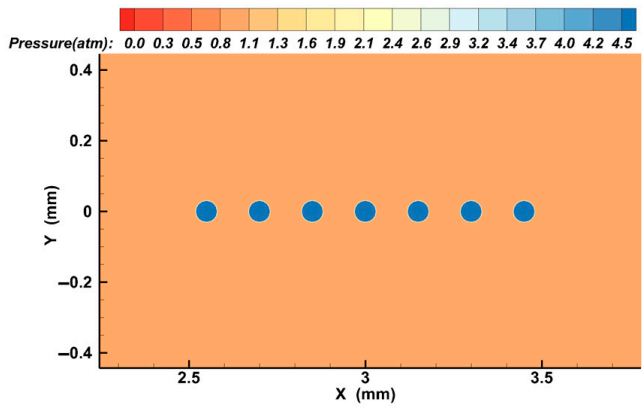

(a) $\mathrm{t}=0$

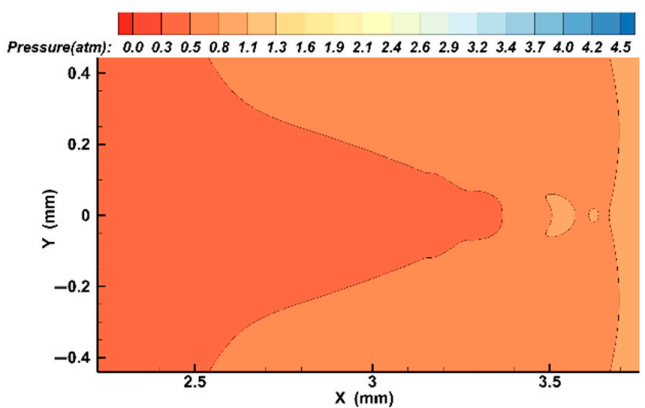

(c) $\mathrm{t}=18 \mu \mathrm{s}$

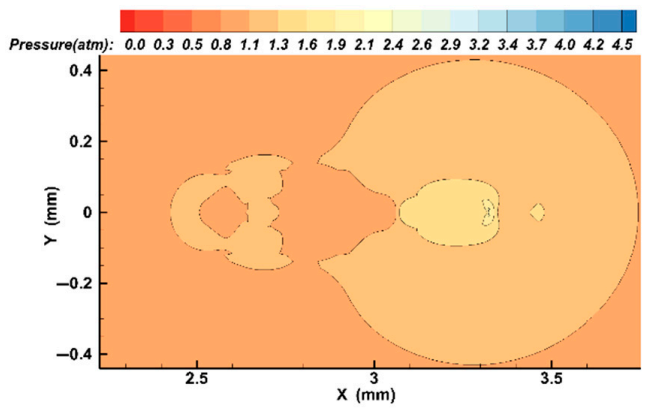

(e) $\mathrm{t}=24 \mu \mathrm{s}$

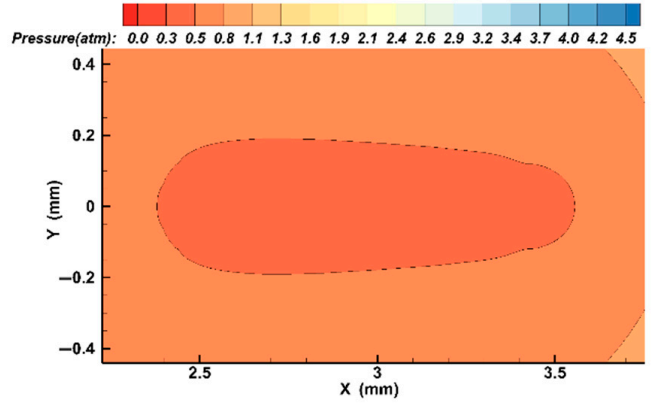

(b) $\mathrm{t}=10 \mu \mathrm{s}$

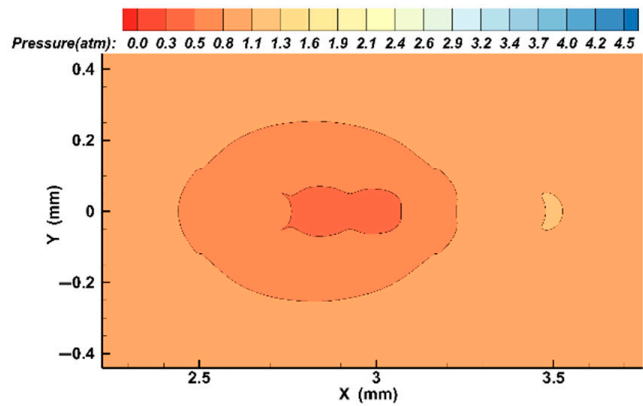

(d) $\mathrm{t}=21 \mu \mathrm{s}$

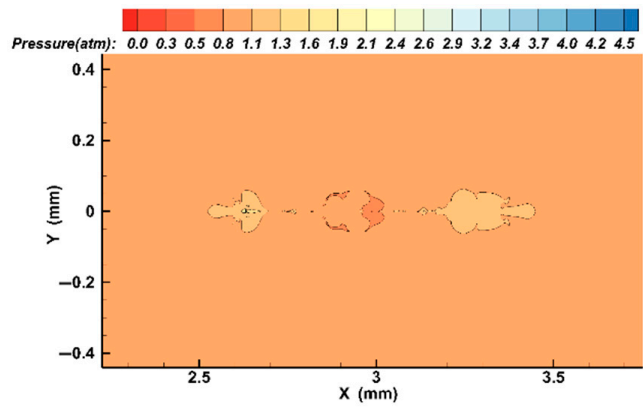

(f) $t=32 \mu \mathrm{s}$

Figure 9. Pressure distribution in the bubble group; $n=7$.

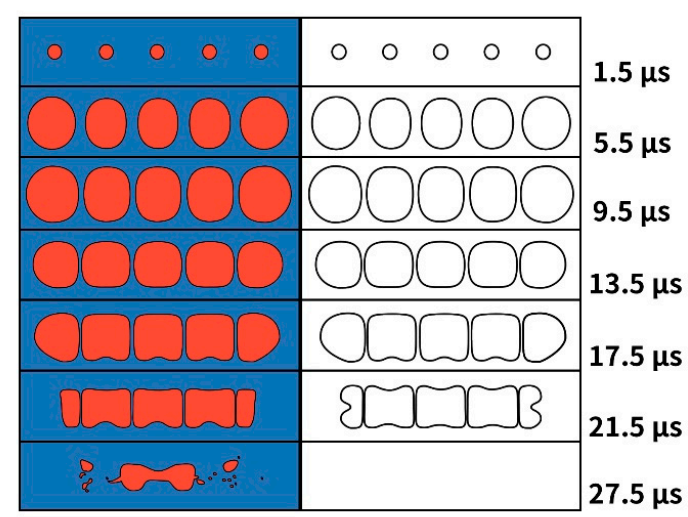

Figure 10. Comparison between experiment and simulation of the cavitation of five bubbles set on a line with $d=200 \mu \mathrm{m}$ and $p_{0}=-1.4 \mathrm{MP}_{\mathrm{a}}, R_{0}=4 \mu \mathrm{m}$ [36].

In order to verify the reliability of the simulation results, the relationship between the first bubble radius and time evolution in the simulation results of Fluent was obtained using the boundary tracking 
method, and the tracking results were compared with the numerical calculation results of bubble motion obtained using Equation (1). The comparison results are shown in Figure 11. The initial radius of the bubbles was $30 \mu \mathrm{m}$ and the distance between bubbles was $150 \mu \mathrm{m}$. Figure 11a shows the evolution curve of the bubble radius with time under different ultrasonic frequencies. Figure $11 \mathrm{~b}$ shows the evolution curve of the bubble radius under different driving ultrasonic intensities. In Figure 11, the lines and points are obtained by Fluent software simulation, and the discrete points are obtained by numerical solution of Equation (1).
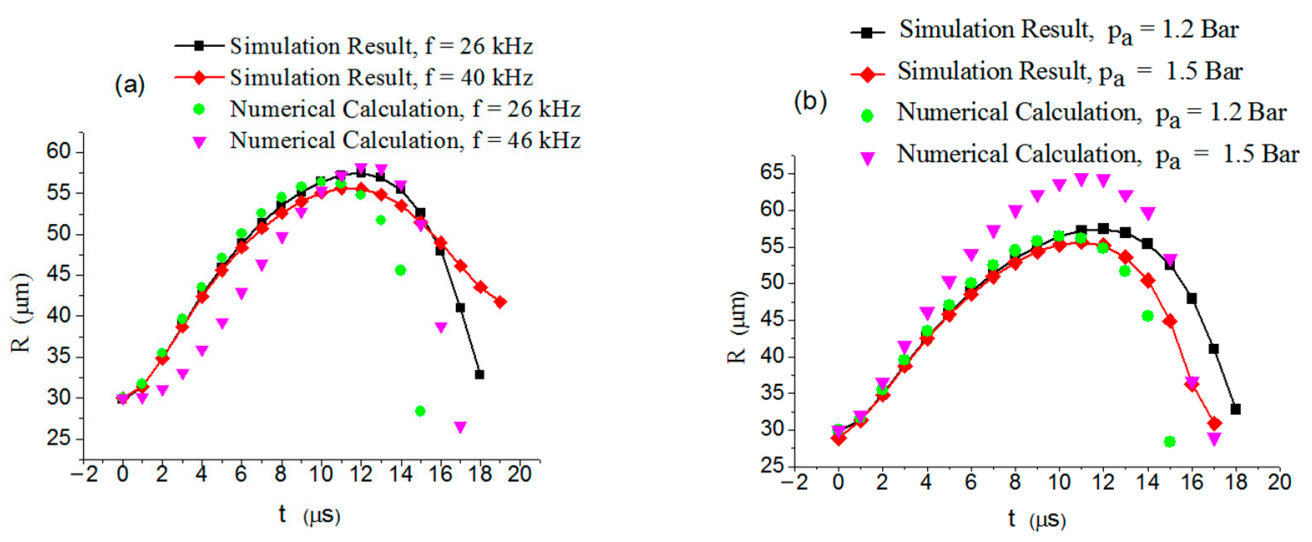

Figure 11. The evolution of bubble radius with time in a columnar bubble group. (a) shows different driving ultrasonic frequencies; the driving ultrasonic frequency is $26 \mathrm{kHz}$ and $40 \mathrm{kHz}, 46 \mathrm{kHz}$. (b) shows different ultrasonic intensities the driving ultrasonic intensity is 1.2Bar and 1.5Bar.

When comparing these curves, it can be seen that the simulation results given by Fluent are consistent with the numerical results obtained using Equation (1) for the bubble growth process, only, in the bubble collapse stage there is a small difference. This difference is caused by the action of a micro-jet, because the influence of a micro-jet cannot be considered in the numerical calculation produced using Equation (1). This shows the reliability of the simulation results. By analyzing the Fluent calculation results, it is further shown that due to the role of the micro-jet, the bubble is broken down by its own micro-jet when the radius is greater than or equal to the initial radius of the bubble. In addition, under the action of different acoustic parameters, bubbles can grow at the same time and reach their maximum radius, but the maximum expansion radius is slightly different. This can be understood as that when the bubble vibrates under the driving of a sound wave, the bubble is not only the sound scattering source but also the transducer in the sound field. When the driving sound intensity increases, the bubble can collect more energy at the same time, meaning the bubble can grow faster and get a larger maximum radius. The process of bubble growth is also the process of energy collection until the micro-jet generated by the collected energy is enough to grasp the bubble, and then the bubble collapses.

\section{Conclusions and Discussion}

In this paper, based on the dynamic equation of bubbles in a bubble group, the resonance frequency of bubbles in a columnar bubble group has been obtained by linear simplification of the bubbles' dynamic equation. The correction coefficient between the resonance frequency of bubbles in a columnar bubble group and the Minnaert frequency of a single bubble has been given. The vibration, growth, and collapse process of five and seven linear bubbles under the action of an ultrasonic field have been simulated by using hydrodynamics software. The results show that:

1. There is a weak coupling interaction between bubbles in the bubble group which can change the resonance frequency of the bubbles. The resonance frequency of bubbles is affected by many factors such as the initial radius of the bubbles, the number of bubbles in a bubble group, and the distance between bubbles. With regard to these parameters, the initial radius of the bubbles 
and the distance between bubbles have the most significant effects on the resonance frequency of the bubbles. For the same number of bubbles, when the distance between the bubbles is larger, the interaction between the bubbles becomes weaker. When the distance between the bubbles increases to 20 times the initial radius of the bubbles, the resonance frequency of the bubbles returns to that of a single bubble. In this case, the interactions between bubbles can be ignored.

2. The first bubble is affected by other bubbles behind it, and the resonance frequency reduces. The larger the number of bubbles, the stronger the interaction force between them, and the smaller the resonance frequency of the bubbles. However, when the number of bubbles increases to twenty and then continues to increase, the number of bubbles and the resonance frequency of the bubbles tends towards a fixed value.

3. Through Fluent simulation results it was found that due to the mutual restriction between bubbles in the expansion process, the energy ratio of the two bubbles at the edge reaches its maximum volume first in the expansion process. Later, the bubbles begin to contract. Through contracting, the bubbles slowly become, and remain, ellipsoid in nature. After this, the two bubbles begin to collapse, forming a micro jet which points to the back of the bubble and along the axis of the center of the linear bubble group, breaking the bubble until it is completely torn apart.

4. The maximum volume and collapse times of bubbles driven by sound parameters are different. The larger the driving frequency and the sound pressure are, the larger the maximum volume of the bubbles.

Author Contributions: Conceptualization, S.-y.L.; methodology, P.-1.Z.; software, P.-1.Z.; writing, P.-1.Z.; writing-review \& editing, S.-y.L.

Funding: This research was funded by the National Natural Science Foundation of China (grant nos. 50875132 and 60573172) and the Industrial Public Relation Project of Shaanxi Technology Committee, China (grant no. 2015GY182).

Conflicts of Interest: The authors declare no conflict of interest.

\section{References}

1. Ryl, J.; Wysocka, J.; Slepski, P.; Darowicki, K. Instantaneous impedance monitoring of synergistic effect between cavitation erosion and corrosion processes. Electrochim. Acta 2016, 203, 388-395. [CrossRef]

2. Mason, T.J.; Lorimer, J.P. Applied Sonochemistry: Uses of Power Ultrasound in Chemistry and Processing; Wiley: Hoboken, NJ, USA, 2002.

3. Bai, L.; Wu, P.; Liu, H.; Yan, J.; Su, C.; Li, C. Rod-shaped cavitation bubble structure in ultrasonic field. Ultrason. Sonochem. 2018, 44, 184-195. [CrossRef] [PubMed]

4. Cui, P.; Zhang, A.M.; Wang, S.P.; Khoo, B.C. Ice breaking by a collapsing bubble. J. Fluid Mech. 2018, 841, 287-309. [CrossRef]

5. Mo, R.Y.; Wang, C.H. Nonlinear acoustic response of two bubble oscillators. Acta Phys. Sin. 2019, 68, 144302.

6. Jiang, L.; Liu, F.; Chen, H.; Wang, J.; Chen, D. Frequency spectrum of the noise emitted by two interacting cavitation bubbles in strong acoustic fields. Phys. Rev. E 2012, 85, 036312. [CrossRef] [PubMed]

7. Mao, Y.; Peng, Y.; Zhang, J. Study of Cavitation Bubble Collapse near a Wall by the Modified Lattice Boltzmann Method. Water 2018, 10, 1439. [CrossRef]

8. Teran, L.A.; Rodriguez, S.A.; Laín, S.; Jung, S. Interaction of particles with a cavitation bubble near a solid wall. Phys. Fluids 2018, 30, 123304. [CrossRef]

9. Jang, N.W.; Gracewski, S.M.; Abrahamsen, B.; Buttaccio, T.; Halm, R.; Dalecki, D. Natural frequency of a gas bubble in a tube: Experimental and simulation results. J. Acoust. Soc. Am. 2009, 126, EL34-EL40. [CrossRef]

10. Sassaroli, E.; Hynynen, K. Forced linear oscillations of micro-bubbles in blood capillaries. J. Acoust. Soc. Am. 2004, 115, 3235-3243. [CrossRef]

11. Wang, C.H.; Lin, S.Y. The nonlinear standing wave inside the space of liquid. Sci. China Phys. Mech. Astron. 2010, 53, 496-503. [CrossRef]

12. Luo, J.; Niu, Z. Jet and Shock Wave from Collapse of Two Cavitation Bubbles. Sci. Rep. 2019, 9, 1352. [CrossRef] [PubMed] 
13. Bremond, N.; Arora, M.; Ohl, C.D.; Lohse, D. Controlled Multibubble Surface Cavitation. Phys. Rev. Lett. 2006, 96, 224501. [CrossRef] [PubMed]

14. Cui, P.; Wang, Q.X.; Wang, S.P.; Zhang, A.M. Experimental study on interaction and coalescence of synchronized multiple bubbles. Phys. Fluids 2016, 28, 012103. [CrossRef]

15. An, Y. Formulation of multibubble cavitation. Phys. Rev. E 2011, 83, 066313. [CrossRef]

16. Ohl, C.D.; Ikink, R. Shock-wave-induced jetting of micron-size bubbles. Phys. Rev. Lett. 2003, 90, 214502. [CrossRef]

17. Tomita, Y.; Shima, A.; Ohno, T. Collapse of multiple gas bubbles by a shock wave and induced impulsive pressure. J. Appl. Phys. 1984, 56, 125. [CrossRef]

18. Han, B.; Köhler, K.; Jungnickel, K.; Mettin, R.; Lauterborn, W.; Vogel, A. Dynamics of laser-induced bubble pairs. J. Fluid Mech. 2015, 771, 706-742. [CrossRef]

19. Futakawa, M. Direct observation and theoretical study of cavitation bubbles in liquid. Phys. Rev. E 2007, 75, 046304.

20. Doinikov, A.A.; Zavtrak, S.T. On the "bubble grapes" induced by a sound field. J. Acoust. Soc. Am. 1998, 99, 3849-3850. [CrossRef]

21. Watanabe, R.; Yanagisawa, K.; Yamagata, T.; Fujisawa, N. Simultaneous shadowgraph imaging and acceleration pulse measurement of cavitating jet. Wear 2016, 358, 72-79. [CrossRef]

22. Moussatov, A.; Granger, C.; Dubus, B. Cone-like bubble formation in ultrasonic cavitation field. Ultrason. Sonochem. 2007, 10, 191-195. [CrossRef]

23. Mettin, R.; Luther, S.; Ohl, C.D.; Lauterborn, W. Acoustic cavitation structures and simulations by a particle model. Ultrason. Sonochem. 1999, 6, 25-29. [CrossRef]

24. Peng-Li, Z.; Shu-Yu, L.; Hua-Ze, Z.; Tao, Z. Coupled resonance of bubbles in spherical cavitation clouds. Acta Phys. Sin. 2019, 68, 134301.

25. Cannon, H.G. On the nature of the centrosomal force. J. Genet. 1923, 13, 47-78. [CrossRef]

26. Ma, Y.; Lin, S.; Xu, J.; Tang, Y. Influnece of nonspherical effects on the secondary Bjerknes force in a strong acoustic field. Acta Phys. Sin. 2017, 66, 014302.

27. König, W.; Ruprecht, E. Effects of convective clouds on the large-scale vorticity budge. Meteorol. Atmos. Phys. 1989, 41, 213-229. [CrossRef]

28. Wang, C.; Hu, J.; Cao, H.; Lin, S.; An, S. Nonlinear resonance of bubble cluster driven by ultrasound. Sci. Sin. Phys. Mech. Astron. 2015, 45, 064301.

29. Spratt, K.S.; Lee, K.M.; Wilson, P. Champagne acoustics. Phys. Today 2018, 71, 66. [CrossRef]

30. Ida, M. Bubble-bubble interaction: A potential source of cavitation noise. Phys. Rev. E 2009, $79,016307$. [CrossRef]

31. Ida, M.; Naoe, T.; Futakawa, M. Suppression of cavitation inception by gas bubble injection: A numerical study focusing on bubble-bubble interaction. Phys. Rev. E 2007, 76, 046309. [CrossRef]

32. An, Y. Nonlinear bubble dynamics of cavitation. Phys. Rev. E 2012, 85, 016305. [CrossRef] [PubMed]

33. Löfstedt, R.; Barber, B.P.; Putterman, S.J. Toward a Hydrodynamic Theory of Sonoluminescence. Phys. Fluids A Fluid Dyn. 1993, 5, 2911-2928. [CrossRef]

34. Barnes, C.W. Euler's Constant and e. Am. Math. Mon. 1984, 91, 428-430. [CrossRef]

35. Guanjun, C. Harmonic series and Euler constant. J. Math. Technol. 1990, 6, 119-121.

36. Bremond, N.; Arora, M.; Dammer, S.M.; Lohse, D. Dammer, Interaction of cavitation bubbles on a wall. Phys. Fluids 2006, 18, 121505. [CrossRef]

37. Abbassi, W.; Besbes, S.; Elhajem, M.; Aissia, H.B.; Champagne, J.Y. Numerical simulation of free ascension and coaxial coalescence of air bubbles using the volume of fluid method (VOF). Comput. Fluids 2018, 161, 47-59. [CrossRef]

(C) 2019 by the authors. Licensee MDPI, Basel, Switzerland. This article is an open access article distributed under the terms and conditions of the Creative Commons Attribution (CC BY) license (http://creativecommons.org/licenses/by/4.0/). 